\title{
Modeling Drivers' Stopping Behaviors during Yellow Intervals at Intersections considering Group Heterogeneity
}

\author{
Juan Li $\mathbb{D}^{1,2}$ Hui Zhang $\mathbb{D D}^{1,2}$ Yanru Zhang $\mathbb{D}^{3}{ }^{3}$ and Xuan Zhang $\mathbb{C}^{4}$ \\ ${ }^{1}$ School of Traffic and Transportation, Beijing Jiaotong University, Beijing, China \\ ${ }^{2}$ Key Laboratory of Transport Industry of Big Data Application Technologies for Comprehensive Transport, \\ Beijing Jiaotong University, Beijing, China \\ ${ }^{3}$ JP Morgan Chase, New York, NY, USA \\ ${ }^{4}$ Sam's Club Technology, Austin, TX, USA
}

Correspondence should be addressed to Juan Li; juanli@bjtu.edu.cn

Received 25 May 2020; Revised 12 June 2020; Accepted 31 October 2020; Published 26 November 2020

Academic Editor: Chunjiao Dong

Copyright (c) 2020 Juan Li et al. This is an open access article distributed under the Creative Commons Attribution License, which permits unrestricted use, distribution, and reproduction in any medium, provided the original work is properly cited.

\begin{abstract}
Stopping behavior during yellow intervals is one of the critical driver behaviors correlated with intersection safety. As the main index of stopping behavior, stopping time is typically described by Accelerated Failure Time (AFT) model. In this study, the comparison of survival curves of stopping time confirms the existence of group specific effects on drivers. However, the AFT model is developed based on the homogeneity assumption. To overcome this drawback, shared frailty survival models are developed for stopping time analysis, which consider the group heterogeneity of drivers. The results show that log-logistic based frailty model with age as a grouping variable has the best goodness of fit and prediction accuracy. Analysis of the models' parameters indicates that phone status, maximum deceleration, vehicles' speed, and the distance to stopping line at the onset of the yellow signal have significant impacts on stopping time. Additionally, heterogeneity analysis illustrates that young, middle-aged, and female drivers are more likely to brake harshly and stop past the stop line, which may block the intersection. Furthermore, drivers, who are more familiar with traffic environments, are more possible to make reasonable stopping decisions approaching intersections. The results can be utilized by traffic authorities to implement road safety strategies, which will help reduce traffic incidents caused by improper stopping behavior at intersections.
\end{abstract}

\section{Introduction}

Signalized intersections are crucial components in road networks, where traffic accidents occur frequently [1]. 15,188 vehicles are involved in fatal crashes at intersections and more than 50 percent of all crashes occurred in intersections according to the statistics on the National Highway Traffic Safety Administration (NHTSA) in 2017 and the Federal Highway Administration (FHWA) in 2018 accordingly $[2,3]$. One of the reasons causing high accident rates at intersections is drivers' incorrect decisions of going or stopping at the onset of a yellow signal. Improper stopping behavior may lead to stopping at intersections illegally, or rear-end collision. Recent research has shown that stopping behavior is a crucial influencing factor for designing traffic facilities, such as red-light camera and countdown timers $[4,5]$, so it is urgent to understand the principle of stopping behavior if we want to reduce the accident rate at intersections.

Most driver behavior studies focus on drivers' decisionmaking processes at the onset of yellow signals, especially in the dilemma zone. Since driving decisions can be seen as a binary classification problem, methods such as binary logistic model $[6,7]$, binary probit model $[8,9]$, and ordered probit model [10] are widely adopted in this issue. In addition, influencing factors on driver behaviors are widely detected, such as vehicle's factors (e.g., speed, deceleration rates, and distance to stop line) [11,12], driver characteristics (e.g., age and gender) $[13,14]$, distracting factors (e.g., cell phone use and listening to music) [15-17]. Results have 
indicated that higher approach speeds are always associated with higher deceleration rates and aggressive driving behavior [12, 18]. Older drivers usually employ greater deceleration levels compared with younger and middle-aged drivers [11], while female drivers generally have a greater level of variability in their driving style [19], and cell phone use has detrimental effects on both novice and experienced drivers [20]. However, the major concern of the above studies is drivers' stop/go decisions, and few studies investigate drivers' stopping behavior approaching intersections.

Driver stopping behavior can be divided into four steps: perception, judgement, manipulation, and stop. Previous research pays more attention to the driver's preparation reaction time (PRT) of braking behavior [21, 22]. Methods commonly focus on the analysis of variance (ANOVA) and a series of linear mixed models [23-25]. However, the stopping process is a continuous-time state, while PRT can only reflect the initial state of the period. Therefore, stopping time is selected to describe the entire stopping behavior at intersections, and the survival analysis model is an appropriate and effective way for timing analysis.

In recent years, the survival analysis model has attracted more attention from researchers as it is convenient and suitable to analyze time-related data $[26,27]$. This model has also been widely used in the transportation field, including traffic accident analysis, travel behavior, pedestrian crossing behavior, and so forth [28-32]. Among survival analysis models, the nonparametric methods and semiparametric models are desirable methods if only the duration times are available or distributional assumptions for the duration data are unexplicit. For example, Tiwari et al. the select nonparametric methods to produce the Kaplan-Meier survival curves analyzing pedestrian risk exposure at signalized intersections [33]. Hao and Cheng conduct nonmotor vehicles' waiting time survival curve to evaluate nonmotor vehicles' street-crossing behavior at signalized crosswalk [34]. However, the above methods cannot build a relationship between event time and key affecting factors, respectively. The parametric AFT duration model is approved by further studies, which can embody specific assumptions about the distribution of failure times. Bella et al. select the AFT model with a Weibull distribution to analyze reaction time and speed reduction time for the evaluation of the effects on driving performance [35]. Li and Silvestri develop an AFT duration model to evaluate the intersection crossing completion time of drivers [36]. Though some key factors were analyzed in these studies, unobserved heterogeneity was neglected for the limitation of homogeneity assumption among objectives in AFT model.

The reasons causing heterogeneity are various, including unobserved covariates, traffic conditions (e.g., traffic volume, vehicles' type, road geometry), and driver characters (e.g., gender, age, and driving experience) [37-39]. Studies have shown that there are clear differences in driving sensitivity, crash severity, and decision-making among different age and gender driver groups when approaching intersections [40-42]. Therefore, it is necessary to consider behavioral differences among driver groups. Methods like random parameters model and latent class model have been combined to develop a mixed logit model and probit model to reflect these differences in driver decision-making behavior [42-44], but these studies mainly focus on unobserved heterogeneity, few studies explore group heterogeneity in stopping behavior at intersections.

Considering the group heterogeneity among driver group, the shared frailty survival model is developed to predict stopping time approaching intersections. The primary contribution of the study is that it makes an initial attempt to avoid the effect of group heterogeneity by incorporating a fragility to describe the difference among driver groups. In this way, the frailty is assumed to be a random distribution across driver groups. Furthermore, the relationship between stopping time and influencing factors has been amply explored. More importantly, the results of the model can be used to make effective traffic management and operational strategies, which will reduce the accidents caused by improper stopping behavior at intersections.

\section{Methodology}

Because the survival analysis model is well-suitable in analyzing the law between events and time, it is employed as a basic model to explore various factors that influence vehicles' stopping time approaching intersections.

2.1. Survival Analysis Model. The survival analysis model has three basic types, nonparametric methods, semiparametric models, and full-parametric models [26]. If there are only the duration times available, then life tables and survival curves can be definitely a good choice to analyze the survival trend for a dataset, so nonparametric methods usually applied for the first step in survival analysis. Semiparametric models, like Cox's proportional hazards model, are suitable for modeling duration data with one or more covariates observed and only minimal assumptions about the underlying distribution. This kind of model is more specific than the nonparametric model undoubtedly, but due to its limited flexibility, it cannot deal with heterogeneity among objectives. Therefore, for further study, more specific and flexible models, parametric models are developed for analyzing survival data.

2.2. AFT Model. AFT model, as a kind of parametric survival analysis model, can mine the impact directly of different influencing factors on survival time and hazard function. In this study, to analyze the different factors that affect the driver's stopping behavior, the timing variable $T$ is defined as the stopping time, which has a probability function $f(t)$, survival function $S(t)$, and hazard function $h(t)$. The survival function is defined as the probability that an individual survives longer than a certain time point $t$ and the hazard function of survival time $T$ gives the conditional failure rate, which is defined as the probability of failure during a small time interval. The specific functions and their relationships are as follows: 


$$
\begin{aligned}
& S(t)=P(T \geq t), \\
& h(t)=\lim _{\Delta t \rightarrow 0} \frac{P(t<T \leq t+\Delta t)}{\Delta t}, \\
& h(t)=\frac{f(t)}{S(t)} .
\end{aligned}
$$

According to the meaning of stopping time, the survival function in this study can reflect the probability of a driver who has not stopped at a certain time point and the hazard function represents the probability of stop during a very small time interval. The greater the hazard rate, the more likely drivers stop at that time point.

The regression form between the log of stopping time $T$ and various variables in the AFT model is as follows:

$$
\log T_{i}=\beta_{0}+\beta_{1} x_{i 1}+\cdots+\beta_{k} x_{i k}+\sigma \varepsilon_{i},
$$

where $\varepsilon_{i}$ is a random disturbance term, $x_{\mathrm{ij}}(j=1,2, \ldots, k)$ represents the $j$ variable of individual $i, \beta_{0}, \ldots, \beta_{k}$ and $\sigma$ are parameters to be estimated, $\beta_{0}$ is the model's constant term, $\beta_{1}, \ldots, \beta_{k}$ is the corresponding coefficient of $x_{\mathrm{ij}}$, and $T_{i}$ represents the survival time of the individual $i$.

One of the advantages of AFT model is that it can provide explicit distributions for stopping time, such as exponential, Weibull, log-normal, log-logistic, and generalized gamma distributions. In this study, the above distributions were all considered in conducting models like equation (2), and the maximum likelihood estimation (MLE) method was used to select the best model.

2.3. Shared Frailty Survival Model. The shared frailty survival model is a type of developed mixture AFT model, which can solve the group heterogeneity among different groups by setting an unobservable multiplicative effect, called frailty, $\alpha$, which is assumed to be random in each cluster $[45,46]$.

For individuals $q\left(l=1, \ldots, n, q=1, \ldots, n_{p}\right)$ in the $l$ group, the hazard function is set as

$$
h_{\mathrm{lq}}\left(t \mid \alpha_{l}\right)=\alpha_{l} h_{\mathrm{lq}}(t)=\alpha_{l} h_{0}(t) \exp \left(\beta^{T} X_{\mathrm{lq}}\right),
$$

where $X_{\mathrm{lq}}$ is the covariate vector and $\beta$ is the fixed coefficient vector to be estimated. $h_{0}(t)$ refers to the baseline hazard function and $h_{\mathrm{lq}}(t)$ represents the individual hazard when the covariate is $X_{\mathrm{lq}}$. The frailty, $\alpha$, is a random positive quantity and, for identifiability of the model, supposed to have mean 1 and variance $\theta$. The larger the variance value, the greater the difference between different driver groups. For mathematical tractability, we limit the choice of frailty distribution to either the gamma $((1 / \theta), \theta)$ distribution or the inverse-Gaussian $(1,(1 / \theta))$ distribution, and the corresponding survival functions are

$$
\text { gamma: } \cdot S_{\theta}(t)=\exp [1-\theta \log \{S(t)\}]^{-(1 / \theta)},
$$

inverse - Gaussian $: \cdot S_{\theta}(t)=\exp \left\{\frac{1}{\theta}\left(1-[2 \theta \log \{S(t)\}]^{(1 / 2)}\right)\right\}$, where $S_{\theta}(t)$ is the survival function under the corresponding frailty distribution and $S(t)$ is the basic survival function.

It can be seen from the hazard function of the shared frailty survival model that the model assumes that all stopping times in a driver group are independent given the frailty, $\alpha$. In other words, the model is a conditionally independent model and mainly deals with differences between different driver groups.

\section{Application to Stopping Time Prediction}

3.1. Data Description and Preparation. The dataset comes from the Driver Behavior Analysis Competition organized by the Transportation Research Board (TRB) in 2014 $[47,48]$. The data study was conducted at the National Advanced Driving Simulator (NADS) at the University of Iowa, which is a high-fidelity driving simulator, aiming to detect the influence of wireless phone use on driver behaviors. During the experiment, each participant had three drives. Each drive consisted of 3 equivalent segments and each segment had one urban field and one rural field. Besides, five signal intersections were set in each segment, and two of the intersections' traffic signals were randomly triggered to change states to yellow when the driver approaches. In addition, secondary tasks were randomly applied, which exposed participants to 3 cell phone interfaces, including baseline (no phone call), outgoing call (calling out), and incoming call (answering a call). For each drive, the participant experienced a different order of segments/ interfaces that are otherwise equivalent. The incoming and outgoing calls were started before the arrival at each segment. Therefore, there were 6 times yellow light decisions for each drive and each participant needed to go through 18 times of that.

The original dataset was collected from 49 participants, with a total of 1157 observations. After deleting the missing and abnormal data, there are still 838 observations left, including 306 "go" observations and 532 "stop" observations. This study selects 532 "stop" observations as the research object.

In this study, seven factors related to driver's stopping behavior were selected as independent variables, including driver's age (Age), gender (Gender), phone status (PS), maximum deceleration (MD), maximum acceleration (MA), vehicle's approaching speed (GTYV), and the distance to the stopping line (GTYD) at the onset of yellow light. The detailed descriptions and values for each variable are shown in Table 1. There are more young drivers in the subject groups and male drivers are a bit more than female, while the numbers of three kinds of phone status' drivers are relatively equal, noticing that maximum decelerations are negative, and the mean of their absolute value is $6.11 \mathrm{~m} \cdot \mathrm{s}^{-2}$. Besides, the mean of the vehicle's speed at the onset of yellow light is $18.95 \mathrm{~m} \cdot \mathrm{s}^{-1}$, and the mean distance is 62.36 meters.

The stopping time (FST) was selected as the duration variable, which was the time from the onset of yellow light to the vehicle stopping completely. Statistics on overall stopping time are shown in Table 2. Vehicles begin to stop at 3.63 seconds after the onset of yellow light and all vehicles stop 
TABLE 1: Statistical description of independent variables.

\begin{tabular}{|c|c|c|c|c|c|}
\hline Variables & Description & Value & Count & Mean & Std. Dev. \\
\hline \multirow{3}{*}{ Age } & Old (50 60 years) & 0 & 131 & & \\
\hline & Middle (30 45 years) & 1 & 197 & & \\
\hline & Young (18 25 years) & 2 & 204 & & \\
\hline \multirow{2}{*}{ Gender } & Female & 0 & 242 & & \\
\hline & Male & 1 & 290 & & \\
\hline \multirow{3}{*}{ PS } & Outcoming call $(O)$ & 0 & 178 & & \\
\hline & Incoming call $(I)$ & 1 & 181 & & \\
\hline & Baseline $(B)$ & 2 & 173 & & \\
\hline $\operatorname{MD}\left(m \cdot s^{-2}\right)$ & Maximum deceleration & Continuous & 532 & -6.11 & 1.53 \\
\hline $\mathrm{MA}\left(\mathrm{m} \cdot \mathrm{s}^{-2}\right)$ & Maximum acceleration & Continuous & 532 & 3.47 & 0.43 \\
\hline $\operatorname{GTYV}\left(\mathrm{m} \cdot \mathrm{s}^{-1}\right)$ & Vehicle's speed at the onset of yellow light & Continuous & 532 & 18.95 & 2.27 \\
\hline GTYD $(\mathrm{m})$ & Distance between the vehicle and the stopping line at the onset of yellow light & Continuous & 532 & 62.36 & 10.56 \\
\hline
\end{tabular}

TABLE 2: Quartile statistics of stopping time.

\begin{tabular}{lcccccccc}
\hline Quantile & $90 \%$ & $75 \%$ & $50 \%$ & $25 \%$ & Min. & Max. & Mean & Std. Dev. \\
\hline Stopping time (s) & 8.92 & 7.68 & 5.84 & 4.69 & 3.63 & 12.97 & 6.24 & 0.078 \\
\hline
\end{tabular}

under 12.97 seconds. The mean stopping time is $6.24 \mathrm{sec}-$ onds and $90 \%, 75 \%, 50 \%$, and $25 \%$ of drivers stop within 8.92 seconds, 7.68 seconds, 5.84 seconds, and 4.69 seconds accordingly.

To understand the probability that vehicles haven't stopped at a certain time better, this study conducts an overall survival curve for 532 "stop" data. Figure 1 presents that vehicles begin to stop at 3.63 seconds and most vehicles can stop completely under 10 seconds.

3.2. Heterogeneity Analysis. Drivers' stopping behaviors may be different under different driver groups; therefore survival curves under different grouping variables were developed for preliminary verification.

The survival curves of stopping time are varied under different driving times, age, gender, and phone states, as shown in Figure 2, which presents evidence for the existence of group heterogeneity. So, in this study, we employ the shared frailty survival model to analyze stopping time, which can avoid the homogeneity assumption of the AFT model.

Behavioral characters of different driver groups are assessed by the dangerous driving index. According to early research, driver behavior is assumed to be statistically consistent within a certain population when facing certain levels of conflicts $[49,50]$. Therefore, this study employs the possibility of dangerous stopping in the original dataset to evaluate the dangerous stopping behavior in different driver groups. The dangerous driving index can be described as the probability of dangerous stopping behaviors and can be calculated:

$$
R=\frac{1}{m} \sum_{r=1}^{m} b_{r}
$$

where $R$ represents the dangerous driving index of each driver group and mrepresents the number of stopping observations in each driver group, when the $r$ observation's record is over-the-line stopping and the value of $b_{r}$ is 1 ; otherwise, $b_{r}$ is set to 0 .

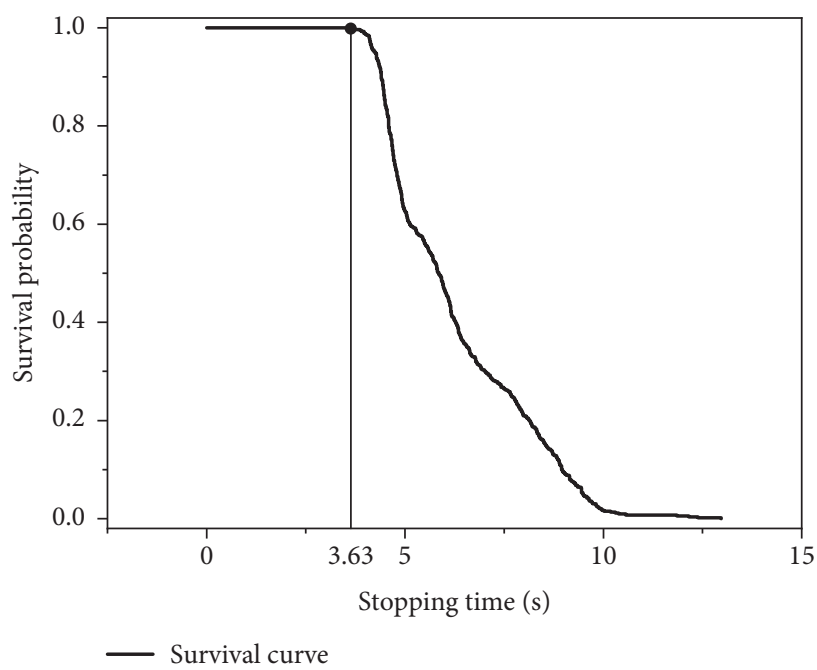

FIgURE 1: Survival curve of overall stopping time.

According to the definition of the dangerous driving index, the greater it is, the greater the tendency of this driver group to stop over the line. Table 3 presents the dangerous driving index for different driver groups. It can be found that the dangerous driving index of middle-aged drivers is the highest among age groups, and young drivers in it are also very high while old drivers' are the lowest. Besides, female drivers' index is higher than male, and the index is a little higher when there are phone interferences than no phone call. The results also show that dangerous driving indexes are changeable under different driving times; especially the index is the highest under the second drive.

\section{Results and Discussion}

4.1. Model Results. Based on 532 "stop" observations approaching intersections, the shared frailty survival models with grouping variables, including driving times, age, gender, and phone status, are constructed, and parameters can 


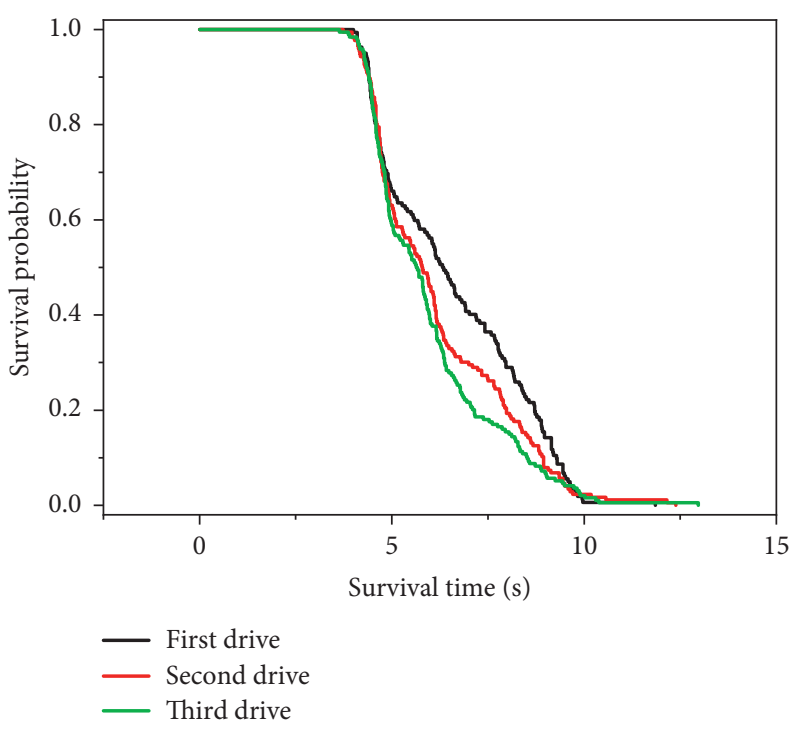

(a)

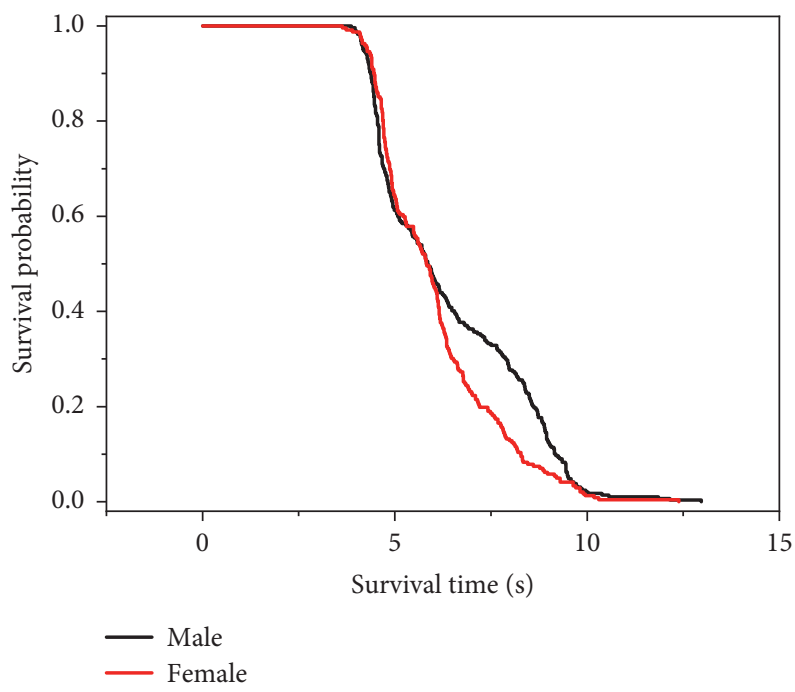

(c)

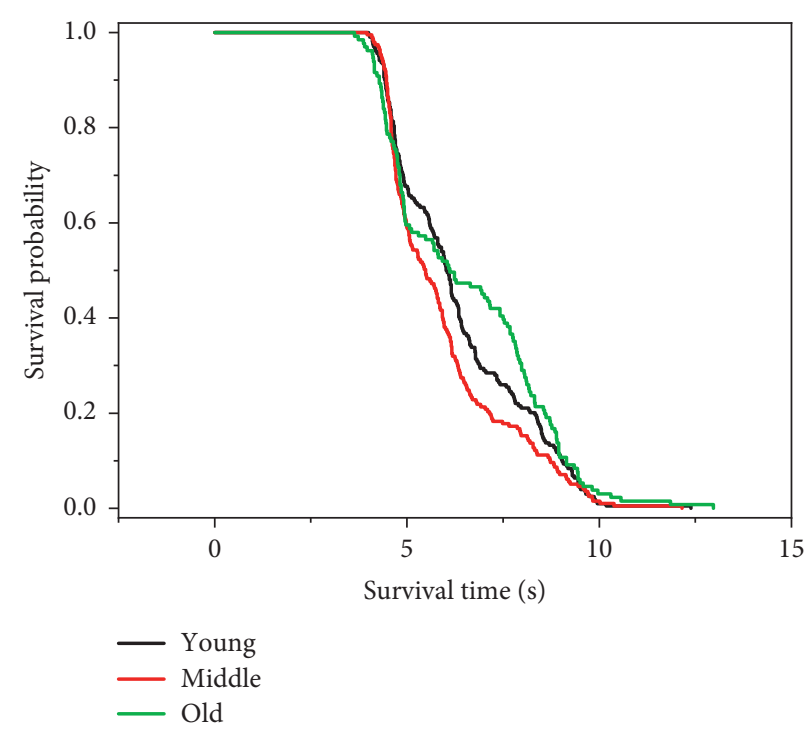

(b)

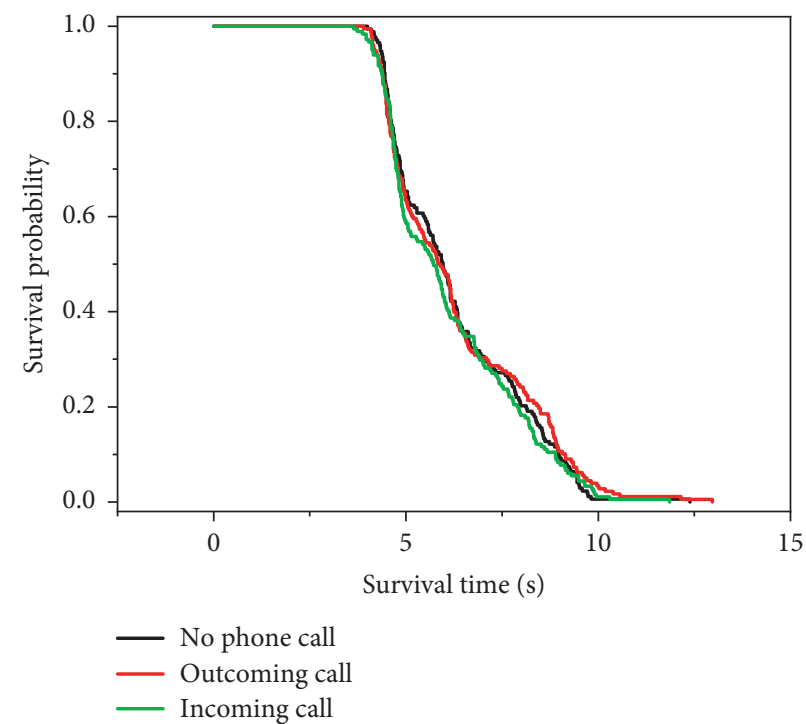

(d)

FIgURE 2: Survival curves under different grouping variables. (a) Survival curves of different driving times. (b) Survival curves of different age groups. (c) Survival curves of different gender groups. (d) Survival curves of different phone status.

TABle 3: Dangerous driving index of different driver groups.

\begin{tabular}{lcccc}
\hline Group & Description & Number of drivers & Number of unsafe stops & Dangerous driving index \\
\hline \multirow{4}{*}{ Age } & Old & 131 & 8 & 0.061 \\
& Middle & 197 & 24 & 0.122 \\
& Young & 204 & 24 & 0.112 \\
\hline \multirow{2}{*}{ Gender } & Female & 242 & 29 & 0.120 \\
& Male & 290 & 27 & 0.093 \\
\hline \multirow{3}{*}{ PS } & Outcoming call & 178 & 19 & 0.107 \\
& Incoming call & 181 & 19 & 0.105 \\
\hline \multirow{3}{*}{ Drive } & Baseline & 173 & 18 & 0.104 \\
& Drive 1 & 162 & 13 & 0.080 \\
& Drive 2 & 176 & 22 & 0.125 \\
\hline
\end{tabular}


be estimated by using the maximum likelihood estimation method at a significance level of 0.1 .

In order to select the best distribution type and grouping variable for modeling the stopping time, two model selection statistics, including the Akaike information criterion (AIC) statistic and the Bayesian information criterion (BIC) statistic are adopted. In general, the model has better goodness of fit with smaller values of the AIC and BIC. Criterion statistics in Table 4 illustrates that generalized gamma distribution is the best-fitted distribution among AFT models, while the log-logistic distribution is proved to be the best distribution among shared frailty survival models, especially when age is selected as grouping variables.

In addition, better distributions are also selected by comparing Cox-Snell residuals [51]. Figures 3(a)-3(d) illustrate the Cox-Snell residuals for different distributions of AFT models; it can be seen that the plotted points in generalized gamma distribution of AFT model fall closer to the reference line with a slope of 1 , which confirms the goodness of fit of the model. At the same time, Figures 3(e)-3(h) indicate that log-logistic distribution is the best fit distribution among shared frailty survival models.

Table 5 presents the estimated results of the parameters of the above good fitted models. Among the shared frailty survival models, except for the model that grouping variable is gender, in which the frailty $\alpha$ follows gamma distribution, other shared frailty survival models' $\alpha$ follow the inverse Gaussian distribution. Variances $\theta$ of frailty $\alpha$ are all far greater than 0 , which also indicate the existence of significant differences between different driver groups. Besides, referring to Table 4, it is easy to find that all shared frailty survival models have smaller values of AIC and BIC than that of the AFT model, which indicates the improved goodness of fit for the shared frailty survival model. Besides, the model with the grouping variable of Age has the smallest value of AIC, BIC. Therefore, the shared frailty survival model with the age grouping variable is selected to analyze the key factors that affect the stopping time.

The results of the optimal model in Table 5 show that phone status, maximum deceleration, vehicle's speed, and the distance to the stopping line at the onset of yellow light are significant variables that affect the stopping time. Different variables' effects on stopping time can be calculated referring to equation (2). Table 6 illustrates the influence of significant variables on the stopping time. It can be seen when incoming calls $(\mathrm{PS}=1)$ that the stopping time is relatively reduced by $1.0 \%\left(e^{-0.001}-1\right)$ compared to outcoming calls $(\mathrm{PS}=0)$ states where the stopping time is increased by $1.3 \%\left(e^{0.013}-1\right)$ compared to no phone calls condition ( $\mathrm{PS}=2)$. Besides, the stopping time under incoming calls is relatively shorter than that of outcoming calls, which means that the driver may stop harshly when receiving a call. The reason for this phenomenon may be that the driver does not have sufficient psychological preparation when receiving a call rather than calling out, so greater distraction is caused, which agrees with the previous research $[20,52]$. At the same time, referring to the dangerous driving index in Table 3 , it is found that the dangerous driving index is higher than no phone call situation when there are phoning tasks, indicating that the driver is more likely to stop over the line when distracted by phone use. Therefore, when approaching intersections, drivers are suggested to avoid distractions such as making and receiving calls. At the same time, because the value of the maximum deceleration (MD) variable in this study is negative, according to the sign of variable coefficients, it is found that the maximum deceleration (MD) and vehicle's speed at the onset of yellow light (GTYV) have a negative impact on the stopping time. When there is a unit increase in the absolute value of maximum deceleration and the value of vehicle speed at the onset of yellow light, the stopping time decreases by $5.8 \%$ and $1.3 \%$ accordingly. Therefore, there is a close relationship between the maximum deceleration, vehicle's speed at the onset of yellow light, and the emergency stop behaviors, so these two factors should be reasonably controlled. Additionally, the distance to the stopping line at the onset of yellow light (GTYD) has a positive effect on the stopping time, and for every meter increase of GTYD, the stopping time increases by $1.1 \%$. Therefore, it is necessary to reasonably control the braking distance to ensure a smooth stopping of the vehicle.

Additionally, other model results in Table 5 also show that Age and Gender variables have an impact on stopping time. The coefficients of young (Age $=2)$, middle (Age $=1$ ), and male $($ Gender $=1)$ drivers are all negative at a significance level; this means young, middle, and female drivers have shorter stopping time and stop more urgently than older and male drivers accordingly, which agree with the previous research finding [19]. Referring to the dangerous driving index in Table 3 , the dangerous driving index of young (0.112) and middle (0.122) drivers is higher than that of old (0.061) drivers, and the index of female drivers (0.120) is higher than that of male drivers (0.093), indicating that young, middle, and female drivers are also more likely to stop over the line, which may cause intersection jams.

4.2. Model Accuracy. The mean absolute percentage error (MAPE) is used to compare the prediction accuracy of the model [53]. The smaller the value, the better the prediction accuracy. The median survival time is selected to calculate the predicted value in this article:

$$
\text { MAPE }=\frac{1}{N} \sum_{i=1}^{n}\left|\frac{y_{i}-y_{i}^{*}}{y_{i}}\right|,
$$

where $N$ is the number of observed individuals, $y_{i}$ is the actual observed value of the individual $i$, and $y_{i}^{*}$ is the predicted value.

Table 7 presents values of MAPE for the AFT model (15.04\%) and the shared frailty survival model with the age grouping variable (13.92\%). It can be seen that both models have a good prediction accuracy [54]. However, compared with the AFT model from a global perspective, there is a negligible improvement in MAPE, $1.12 \%$, in the shared frailty survival model. Therefore, this study adopts a categorical analysis in prediction accuracy. Note that $75 \%$ of drivers will stop under 7.7 seconds in Table 2, so this study 
Table 4: Statistics of criterion based on different distribution types.

\begin{tabular}{lcccccccc}
\hline \multirow{2}{*}{ Criterion } & \multicolumn{4}{c}{ AFT model distribution type } & \multicolumn{4}{c}{ Shared frailty survival model distribution type } \\
& Weibull & Log-normal & Log-logistic & Generalized gamma & Loglogistic (d-rive) & Log-logistic (age) & Log-logistic (gender) & Log-logistic (PS) \\
\hline AIC & 90 & -88 & -101 & $\mathbf{- 5 0 8}$ & -517 & $\mathbf{- 5 2 6}$ & -529 & -500 \\
BIC & 146 & -33 & -46 & $\mathbf{- 4 4 8}$ & -474 & $\mathbf{- 4 9 1}$ & -482 & -457 \\
\hline
\end{tabular}

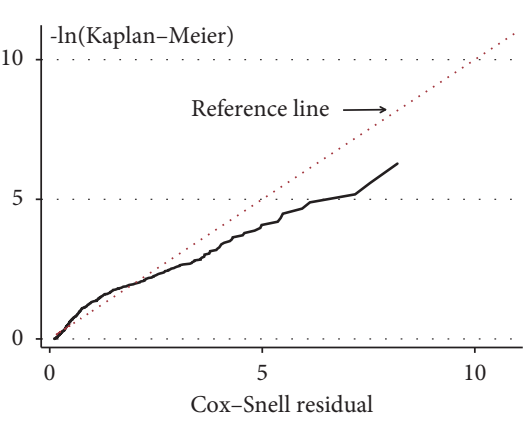

(a)

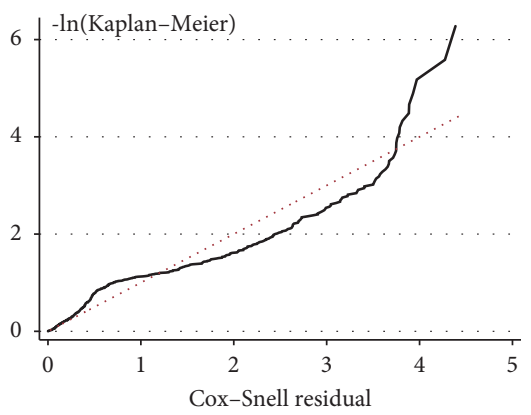

(d)

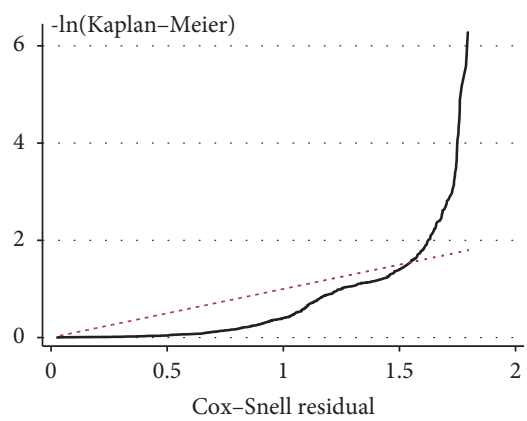

(g)

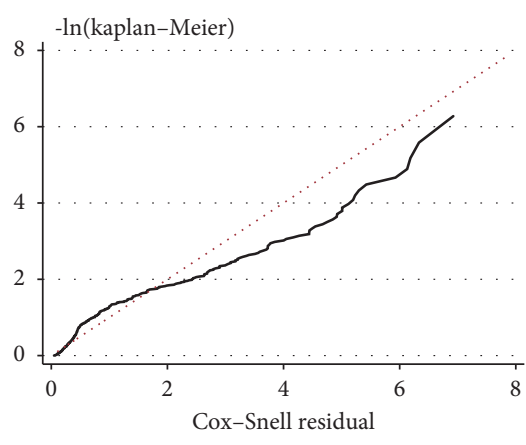

(b)

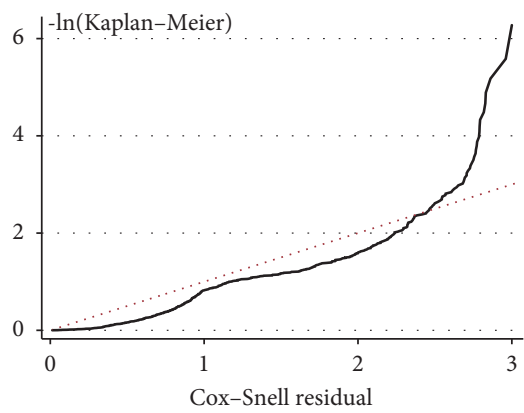

(e)

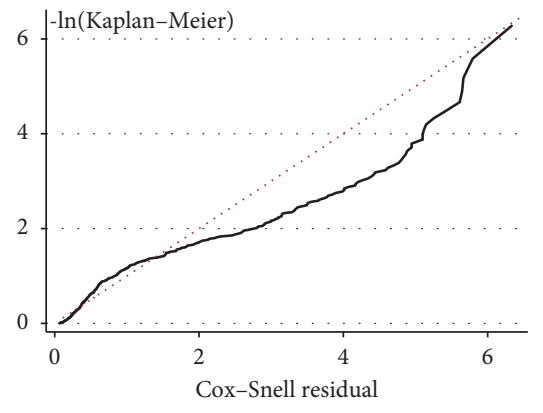

(c)

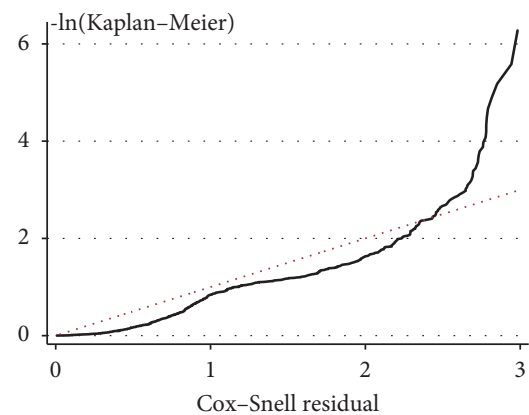

(f)

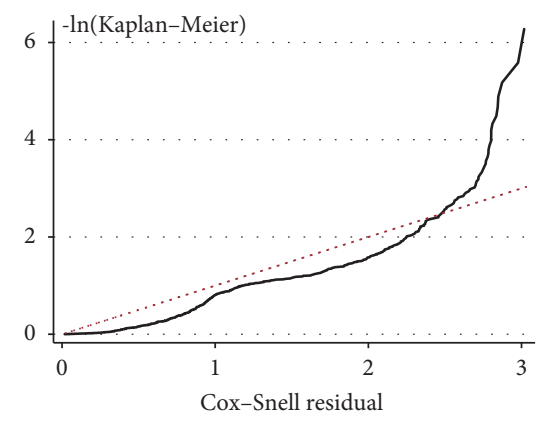

(h)

Figure 3: Cox-Snell residuals for different distribution types.(a) Weibull. (b) Log-normal. (c) Log-logistic. (d) General gamma. (e) Loglogistic (Drive). (f) Log-logistic (Age). (g) Log-logistic (Gender). (h) Log-logistic (PS).

divides the dataset into two categories at this time point and analyzes the prediction accuracy for each data category. Table 7 presents the accuracy results.

When the stopping time is less than 7.7 seconds, the improved shared model can have a very high accurate prediction (MAPE is $6 \%$ ) and a small mean prediction error $(0.36 \mathrm{sec})$; besides, it improves prediction accuracy by $3 \%$ compared with AFT model. Though both the above models do not have good prediction accuracy when stopping time is larger than 7.7 seconds, the prediction accuracy is also acceptable [54]. Therefore, the predictions can be used to analyze driver stopping behaviors reasonably.

4.3. Effects of Group Heterogeneity. As mentioned above, the evidence of heterogeneity is seen in the core results of the models presented in Figure 2 and Table 5. It is found that, compared with the AFT model, the shared frailty survival model's advantages are reflected not only in improving the model's goodness of fitting and prediction accuracy but also 
Table 5: Model estimation results.

\begin{tabular}{|c|c|c|c|c|c|}
\hline \multirow{2}{*}{ Variables } & \multirow{2}{*}{ AFT model } & \multicolumn{4}{|c|}{ Shared frailty survival model } \\
\hline & & Group variable (drive) & Group variable (age) & Group variable (gender) & Group variable (PS) \\
\hline Distribution of FST & Generalized gamma & Log-logistic & Log-logistic & Log-logistic & Log-logistic \\
\hline Distribution of $\alpha$ & - & Inverse Gaussian & Inverse Gaussian & Gamma & Inverse Gaussian \\
\hline Variable & Coefficient & Coefficient & Coefficient & Coefficient & Coefficient \\
\hline Age $=1$ & -0.005 & -0.007 & - & -0.007 & -0.006 \\
\hline Age $=2$ & $-0.017^{* * *}$ & $-0.013^{* *}$ & - & $-0.011^{*}$ & $-0.014^{* *}$ \\
\hline Gender $=1$ & - & - & - & $-0.014^{* * *}$ & - \\
\hline $\mathrm{PS}=1$ & 0.004 & 0.001 & -0.001 & -0.002 & 0.004 \\
\hline $\mathrm{PS}=2$ & $0.021^{* * *}$ & $0.016^{* * *}$ & $0.013^{* *}$ & $0.012^{* *}$ & $0.019^{* * *}$ \\
\hline MD & $0.056^{* * *}$ & $0.056^{* * *}$ & $0.056^{* * *}$ & $0.055^{* * *}$ & $0.056^{* * *}$ \\
\hline MA & - & - & - & - & - \\
\hline GTYV & $-0.014^{* * *}$ & $-0.013^{* * *}$ & $-0.013^{* * *}$ & $-0.013^{* * *}$ & $-0.013^{* * *}$ \\
\hline GTYD & $0.011^{* * *}$ & $0.011^{* * *}$ & $0.011^{* * *}$ & $0.011^{* * *}$ & $0.011^{* * *}$ \\
\hline Constant & $1.511^{* * *}$ & $1.483^{* * *}$ & $1.472^{* * *}$ & $1.477^{* * *}$ & $1.481^{* * *}$ \\
\hline$\theta$ & - & 31.273 & 27.512 & 3.380 & 32.601 \\
\hline
\end{tabular}

$\theta$ is the variance of frailty $\alpha .{ }^{* * *},{ }^{* *},{ }^{*}$ mean statistical significance at $\alpha=0.01,0.05,0.1$, respectively.

TABLE 6: Driver's stopping risk percentage change.

\begin{tabular}{lccc}
\hline Variable & Coefficient & EXP $(\beta)$ & Percentage change $(\%)$ \\
\hline PS $=1$ & -0.001 & 0.999 & -1.0 \\
PS $=2$ & 0.013 & 1.013 & 1.3 \\
MD & 0.056 & 1.058 & 5.8 \\
GTYV & -0.013 & 0.987 & -1.3 \\
GTYD & 0.011 & 1.011 & 1.1 \\
\hline
\end{tabular}

TABLE 7: Statistics on model accuracy.

\begin{tabular}{|c|c|c|c|c|}
\hline Stopping time category $(\mathrm{sec})$ & Count & Model & MAPE (\%) & Mean prediction error $(\mathrm{sec})$ \\
\hline & & AFT & 15.04 & 1.11 \\
\hline Overall & 532 & Shared (Age) & 13.92 & 1.13 \\
\hline $\mathrm{FST} \leq 7.7$ & $400(75 \%)$ & AFT & 9 & 0.51 \\
\hline $\mathrm{FS1}=1.7$ & $400(/ 5 \%)$ & Shared (Age) & 6 & 0.36 \\
\hline FST $>7.7$ & $132(25 \%)$ & $\begin{array}{c}\text { AFT } \\
\text { Shared (Age) }\end{array}$ & $\begin{array}{l}32 \\
38\end{array}$ & $\begin{array}{l}2.94 \\
3.46\end{array}$ \\
\hline
\end{tabular}

in the mining impacts of other potential variables on drivers' stopping behaviors.

Comparing with the AFT model, the value and sign of significant variables' coefficients have changed in the shared frailty survival model. For example, in the shared frailty survival model with grouping variable age, the sign of incoming calls' $(\mathrm{PS}=1)$ coefficient changes to negative compared to the AFT model, which is more in line with the fact that the incoming distraction situation has a greater impact on the driver's behavior. What is more, the reduced absolute value of coefficients for phoning states and vehicle's speed at the onset of yellow light variables, reduced constant terms of all shared frailty survival models compared to the AFT model, also illustrates that some of the significant variables' effect on stopping time is shared after considering the heterogeneity of varied driver groups. Besides, other shared frailty survival models also highlight the significant effects caused by age, gender, and different driving times on drivers' stopping behavior. Table 3 shows that, in the first, second, and third drives, the dangerous driving indexes show a trend of increasing first and then decreasing. The dangerous driving index in the second drive is the highest with a value of 0.125 . The reason for this phenomenon may be drivers in the second drive have been already familiar with the driving environment and are not concentrating on driving enough. While in the third drive, drivers accumulate the previous two driving experiences and make the stopping decision more accurate and reduced the probability of stopping over the line, which reflects that familiarity with the traffic environment directly affects the safety of driver's stopping behavior, drivers who are more familiar with the traffic environment can make more reasonable stopping decisions, and these effects are difficult to mine by the AFT model.

\section{Conclusions}

To further understand drivers' stopping behaviors at intersections during the yellow interval, a survival analysis model was proposed. However, the parameter AFT model has a homogeneity assumption among drivers. To overcome this limitation, the developed shared frailty survival models 
with grouping variables, including driving times, age, gender, and phone status, were conducted in this study.

Results show that the shared frailty survival models can improve the fitness and prediction accuracy compared with the AFT model, and the model with the grouping variable of age fits best. In this best-fitting model, variables, including phone status, maximum deceleration, vehicle's speed, and the distance to the stopping line at the onset of yellow light, have significant effects on stopping time. Drivers stop relatively in emergency and are easier to stop over the line when there are phoning tasks' distraction. The greater the vehicle's speed at the onset of yellow light and the absolute value of the maximum deceleration, the more likely the vehicle to have an emergency stop, while the greater the value of the distance to the stopping line at the onset of yellow light, the smoother the vehicle stop.

By heterogeneity analysis, other potential influencing factors on stopping time are also explored in this study. Except for the best-fitting shared frailty survival model, models with other grouping variables also highlight the driving behaviors' significant differences in varied age, gender, and driving times groups. Results show that young, middle, and female drivers are more likely to stop emergency and stop over the line, which may cause intersection jams. Besides, familiarity with the traffic environment has a direct impact on the safety of the stopping behavior; drivers who more familiar with the traffic condition could make a more reasonable stopping decision.

Based on the above results, drivers are recommended to reasonably predict the braking distance when approaching the signalized intersections, control the vehicle's speed and deceleration, and avoid distracting behaviors such as having phone calls. Besides, young, middle-aged, and female drivers are more likely to stop over the stop line and block the intersections. These groups of people may need more attention from traffic managers such as intensive observation. Familiarity with the traffic environment may help drivers make proper decisions, so intersection safety will be improved if drivers are provided useful road and traffic information such as speed limits and signal ahead. However, the dataset in this study only contains three driving times, so related data collection is needed for further studying the explicit effects of the familiarity with traffic environment on drivers' stopping behaviors. This study can help researchers better understand driver stopping behavior at the onset of yellow light and also can be applied to driver assistance systems and intersection design studies.

\section{Data Availability}

The data used for this study were taken from experiments conducted at the University of Iowa-National Advanced Driving Simulator (NADS). The raw data are attached, which can be accessed from the website: http://depts. washington.edu/hfsm/upload.php.

\section{Conflicts of Interest}

The authors declare that there are no conflicts of interest regarding the publication of this paper.

\section{Acknowledgments}

This research was supported by the Fundamental Research Funds for the Central Universities (no. 2019JBM036).

\section{Supplementary Materials}

Our processed stopping data are attached. (Supplementary Materials)

\section{References}

[1] H. Huang, D. Wang, L. Zheng, and X. Li, "Evaluating timereminder strategies before amber: common signal, green flashing and green countdown," Accident Analysis \& Prevention, vol. 71, pp. 248-260, 2014.

[2] National Highway Traffic Safety Administration, "Traffic safety facts 2017 a compilation of motor vehicle crash data (annual report)," 2017, https://crashstats.nhtsa.dot.gov/Api/ Public/ViewPublication/812806.

[3] The Federal Highway Administration (FHWA), "Intersection safety: background and objectives," 2018, https://highways. dot.gov/research-programs/safety/intersection-safety.

[4] F. Baratian-Ghorghi, H. Zhou, and A. Franco-Watkins, "Effects of red light cameras on driver's stop/go decision: assessing the green extension hypothesis," Transportation Research Part F: Traffic Psychology and Behaviour, vol. 46, pp. 87-95, 2017.

[5] M. Huang, M. Fujita, and W. Wisetjindawat, "Countdown timers, video surveillance and drivers' stop/go behavior: winter versus summer," Accident Analysis \& Prevention, vol. 98, pp. 185-197, 2017.

[6] H. Köll, M. Bader, and K. W. Axhausen, "Driver behaviour during flashing green before amber: a comparative study," Accident Analysis \& Prevention, vol. 36, no. 2, pp. 273-280, 2004.

[7] T. J. Gates, D. A. Noyce, L. Laracuente, and E. V. Nordheim, "Analysis of driver behavior in dilemma zones at signalized intersections," Transportation Research Record: Journal of the Transportation Research Board, vol. 2030, no. 1, pp. 29-39, 2007.

[8] Y. Sheffi and H. Mahmassani, "A model of driver behavior at high speed signalized intersections," Transportation Science, vol. 15 , no. 1, pp. 50-61, 1981.

[9] P. Li and M. Abbas, "Stochastic dilemma hazard model at high-speed signalized intersections," Journal of Transportation Engineering, vol. 136, no. 5, pp. 448-456, 2010.

[10] Y. Liu, G.-L. Chang, and J. Yu, "Empirical study of driver responses during the yellow signal phase at six Maryland intersections," Journal of Transportation Engineering, vol. 138, no. 1, pp. 31-42, 2012.

[11] I. El-Shawarby, H. Rakha, A. Amer, and C. McGhee, "Impact of driver and surrounding traffic on vehicle deceleration behavior at onset of yellow indication," Transportation Research Record: Journal of the Transportation Research Board, vol. 2248, no. 1, pp. 10-20, 2011.

[12] J. Wang, K. K. Dixon, H. Li, and J. Ogle, "Normal deceleration behavior of passenger vehicles at stop sign-controlled intersections evaluated with in-vehicle global positioning system data," Transportation Research Record: Journal of the Transportation Research Board, vol. 1937, no. 1, pp. 120-127, 2005.

[13] C. Y. D. Yang and W. G. Najm, "Examining driver behavior using data gathered from red light photo enforcement 
cameras," Journal of Safety Research, vol. 38, no. 3, pp. 311-321, 2007.

[14] J. J. Lu and J. C. Pernía, "The differences of driving behavior among different driver age groups at signalized intersections," IATSS Research, vol. 24, no. 2, pp. 75-84, 2000.

[15] R. M. Brumfield and S. S. Pulugurtha, "Effect of driver cell phone use on queue discharge patterns at signalized intersections," Transportation Research Record: Journal of the Transportation Research Board, vol. 2257, no. 1, pp. 71-79, 2011.

[16] M. E. Rakauskas, L. J. Gugerty, and N. J. Ward, "Effects of naturalistic cell phone conversations on driving performance," Journal of Safety Research, vol. 35, no. 4, pp. 453-464, 2004.

[17] A. K. Huemer, M. Schumacher, M. Mennecke, and M. Vollrath, "Systematic review of observational studies on secondary task engagement while driving," Accident Analysis \& Prevention, vol. 119, pp. 225-236, 2018.

[18] H. Rakha, A. Amer, and I. El-Shawarby, "Modeling driver behavior within a signalized intersection approach decisiondilemma zone," Transportation Research Record: Journal of the Transportation Research Board, vol. 2069, no. 1, pp. 16-25, 2008.

[19] S. M. Lavrenz, V. Dimitra Pyrialakou, and K. Gkritza, "Modeling driver behavior in dilemma zones: a discrete/ continuous formulation with selectivity bias corrections," Analytic Methods in Accident Research, vol. 3-4, pp. 44-55, 2014.

[20] J. Cooper, C. Yager, and S. T. Chrysler, "An investigation of the effects of reading and writing text-based messages while driving," Southwest Region University Transportation Center, College Station, TX, USA, No. SWUTC/11/476660-00024-1, 2011.

[21] Y. D. Wong and P. K. Goh, "Driver perception-response time for braking action during signal change interval," Road Transport Research, vol. 9, pp. 17-26, 2000.

[22] P.-K. Goh and Y.-D. Wong, "Driver perception response time during the signal change interval," Applied Health Economics and Health Policy, vol. 3, no. 1, pp. 9-15, 2004.

[23] C. Ariën, E. M. M. Jongen, K. Brijs, T. Brijs, S. Daniels, and G. Wets, "A simulator study on the impact of traffic calming measures in urban areas on driving behavior and workload," Accident Analysis \& Prevention, vol. 61, pp. 43-53, 2013.

[24] Q. Hussain, A. Pirdavani, C. Arien, T. Brijs, and W. K. M. Alhajyaseen, "The impact of perceptual countermeasures on driving behavior in rural-urban transition road segments: a driving simulator study," Advanced Transportation Stud.: International Journal, vol. 46, pp. 83-96, 2018.

[25] Q. Hussain, W. K. M. Alhajyaseen, K. Brijs, A. Pirdavani, and T. Brijs, "Innovative countermeasures for red light running prevention at signalized intersections: a driving simulator study," Accident Analysis \& Prevention, vol. 134, Article ID 105349, 2020.

[26] S. P. Washington, M. G. Karlaftis, and F. L. Mannering, Statistical and Econometric Methods for Transportation Data Analysis, Chapman \& Hall/CRC, Boca Raton, FL, USA, 2nd ed. edition, 2011.

[27] D. A. Hensher and F. L. Mannering, "Hazard-based duration models and their application to transport analysis," Transport Reviews, vol. 14, no. 1, pp. 63-82, 1994.

[28] J. Weng, Y. Zheng, X. Yan, and Q. Meng, "Development of a subway operation incident delay model using accelerated failure time approaches," Accident Analysis \& Prevention, vol. 73, pp. 12-19, 2014.
[29] X. Yang, Z. Gao, H. Guo, and M. Huan, "Survival analysis of car travel time near a bus stop in developing countries," Science China Technological Sciences, vol. 55, no. 8, pp. 2355-2361, 2012.

[30] A. Mohammadian and S. T. Doherty, "Modeling activity scheduling time horizon: duration of time between planning and execution of pre-planned activities," Transportation Research Part A: Policy and Practice, vol. 40, no. 6, pp. 475-490, 2006.

[31] P. Van den Berg, T. Arentze, and H. Timmermans, "A latent class accelerated hazard model of social activity duration," Transportation Research Part A: Policy and Practice, vol. 46, no. 1, pp. 12-21, 2012.

[32] X. Yang, M. Abdel-Aty, M. Huan, Y. Peng, and Z. Gao, "An accelerated failure time model for investigating pedestrian crossing behavior and waiting times at signalized intersections," Accident Analysis \& Prevention, vol. 82, pp. 154-162, 2015.

[33] G. Tiwari, S. Bangdiwala, A. Saraswat, and S. Gaurav, "Survival analysis: pedestrian risk exposure at signalized intersections," Transportation Research Part F: Traffic Psychology and Behaviour, vol. 10, no. 2, pp. 77-89, 2007.

[34] P. Hao and W. Cheng, "Nonparametric survival analysis of non-motor vehicles violation in signal intersection," Applied Mechanics and Materials, vol. 713-715, pp. 2115-2118, 2015.

[35] F. Bella and M. Silvestri, "Effects of directional auditory and visual warnings at intersections on reaction times and speed reduction times," Transportation Research Part F: Traffic Psychology and Behaviour, vol. 51, pp. 88-102, 2017.

[36] X. Li, O. Oviedo-Trespalacios, and A. Rakotonirainy, “Drivers' gap acceptance behaviours at intersections: a driving simulator study to understand the impact of mobile phone visualmanual interactions," Accident Analysis \& Prevention, vol. 138, Article ID 105486, 2020.

[37] A. B. Ellison, S. Greaves, and M. Bliemer, "Examining heterogeneity of driver behavior with temporal and spatial factors," Transportation Research Record: Journal of the Transportation Research Board, vol. 2386, no. 1, pp. 158-167, 2013.

[38] P. Maheshwary, K. Bhattacharyya, B. Maitra, and M. Boltze, "A methodology for calibration of traffic micro-simulator for urban heterogeneous traffic operations," Journal of Traffic and Transportation Engineering, vol. 7, no. 4, 2019.

[39] B. K. Pathivada and V. Perumal, "Modeling driver behavior in dilemma zone under mixed traffic conditions," Transportation Research Procedia, vol. 27, pp. 961-968, 2017.

[40] S. Hong, B. Min, S. Doi, and K. Suzuki, "Approaching and stopping behaviors to the intersections of aged drivers compared with young drivers," International Journal of Industrial Ergonomics, vol. 54, pp. 32-41, 2016.

[41] A. Rakotonirainy, D. Steinhardt, P. Delhomme, M. Darvell, and A. Schramm, "Older drivers' crashes in Queensland, Australia," Accident Analysis \& Prevention, vol. 48, pp. 423429, 2012.

[42] P. T. Savolainen, "Examining driver behavior at the onset of yellow in a traffic simulator environment: comparisons between random parameters and latent class logit models," Accident Analysis \& Prevention, vol. 96, pp. 300-307, 2016.

[43] P. Seraneeprakarn, S. Huang, V. Shankar, F. Mannering, N. Venkataraman, and J. Milton, "Occupant injury severities in hybrid-vehicle involved crashes: a random parameters approach with heterogeneity in means and variances," $A n$ alytic Methods in Accident Research, vol. 15, pp. 41-55, 2017. 
[44] P. T. Savolainen, A. Sharma, and T. J. Gates, "Driver decisionmaking in the dilemma zone-examining the influences of clearance intervals, enforcement cameras and the provision of advance warning through a panel data random parameters probit model," Accident Analysis \& Prevention, vol. 96, pp. 351-360, 2016.

[45] P. K. Andersen, J. P. Klein, K. M. Knudsen, and R. T. Palacios, "Estimation of variance in cox's regression model with shared gamma frailties," Biometrics, vol. 53, no. 4, pp. 1475-1484, 1997.

[46] H. T. V. Vu, "Estimation in semiparametric conditional shared frailty models with events before study entry," Computational Statistics \& Data Analysis, vol. 45, no. 3, pp. 621637, 2004.

[47] Transportation Research Board, "2014 TRB data competition," 2014, http://trbstats.weebly.com/2014-trb-datacompetition.html.

[48] The National Advanced Driving Simulator, 2014, http://www. nads-sc.uiowa.edu/sim_nads1.php.

[49] X. Zou and D. M. Levinson, "Modeling pipeline driving behaviors," Transportation Research Record: Journal of the Transportation Research Board, vol. 1980, no. 1, pp. 16-23, 2006.

[50] J. Li, Q. He, H. Zhou, Y. Guan, and W. Dai, "Modeling driver behavior near intersections in hidden markov model," International Journal of Environmental Research and Public Health, vol. 13, no. 12, p. 1265, 2016.

[51] J. Weng, W. Qiao, X. Qu, and X. Yan, "Cluster-based lognormal distribution model for accident duration," Transportmetrica A: Transport Science, vol. 11, no. 4, pp. 345-363, 2015.

[52] M. M. Haque, A. D. Ohlhauser, S. Washington, and L. N. Boyle, "Decisions and actions of distracted drivers at the onset of yellow lights," Accident Analysis \& Prevention, vol. 96, pp. 290-299, 2016.

[53] Q. Meng and X. Qu, "Estimation of rear-end vehicle crash frequencies in urban road tunnels," Accident Analysis \& Prevention, vol. 48, pp. 254-263, 2012.

[54] Y. Chung, "Development of an accident duration prediction model on the Korean freeway systems," Accident Analysis \& Prevention, vol. 42, no. 1, pp. 282-289, 2010. 